\title{
iDASH secure genome analysis competition 2017
}

\author{
XiaoFeng Wang ${ }^{1 *}$, Haixu Tang ${ }^{1 \dagger}$, Shuang Wang ${ }^{2 \dagger}$, Xiaogian Jiang ${ }^{3+}$, Wenhao Wang ${ }^{1}$, Diyue Bu ${ }^{1}$, Lei Wang ${ }^{1}$, \\ Yicheng Jiang ${ }^{2}$ and Chenghong Wang ${ }^{2}$
}

From iDASH Privacy and Security Workshop 2017

Orlando, FL, USA. 14 October 2017

Year 2017 marks the 4th anniversary since the first iDASH Secure Genome Analysis Competition [1] launched jointly by University California San Diego and Indiana University, Bloomington. The past 4 years have witnessed the continued progresses in genomic and biomedical technologies, with their influence permeating our daily life, redefining our perception of privacy. As an example, when the law enforcement identified the golden state killer from his remote relative's DNA from GEDmatch [2], the kinship inference attack reported 3 years ago [3] now becomes reality. Facing the genome privacy community are still these old devils: protecting the privacy during genomic data sharing and genome analysis. Just they look increasingly real every day, which mounts a great pressure on the young community to come up with practical, usable solutions.

Seeking such practical privacy solutions has always been at the center of the competition, as set by the organizers 4 years ago. This year, serving the purpose are three carefully designed competition tracks, including De-duplication for GA4GH (Track 1), SGX-based whole genome variation search (Track 2) and HME based logistic regression model learning (Track 3). Each track either has its root in the real privacy problem haunting the already deployed system or new challenges emerged from innovative applications of new computing techniques to support secure genomic data sharing or analysis.

Specifically, in collaboration with the Global Alliance for Genomics and Health (GA4GH), Track 1 looks for new privacy-preserving patient linkage (PPRL) solution for removing duplicated health records maintained by

\footnotetext{
* Correspondence: xw7@indiana.edu

†Xiao Feng Wang, Haixu Tang, Shuang Wang and Xiaoqian Jiang contributed equally to this work.

${ }^{1} S c h o o l$ of Informatics, Computing and Engineering, Indiana University,

Bloomington, IN 47408, USA

Full list of author information is available at the end of the article
}

multiple data owners. The new solution can be applied on top of existing European ENCCA unified patient identifier framework to facilitate record deduplication in GA4GH. Track 2 is meant to seek new answers for a long-standing genome privacy problem: how to perform a large-scale Genome-Wide Association Study (GWAS) on the untrusted public cloud. This time, however, the attempts are made by leveraging Intel's Software Guard Extension (SGX), a new hardware trusted execution environment (TEE) support, to move a secure analysis solution closer to practical use. Track 3 is designed to answer the new demand for training a machine learning model (logistic regression) on encrypted genomic data, when the computation needs to be conducted in an untrusted environment, through a homomorphic encryption (HME) scheme.

Altogether, these three tracks attracted 65 participation teams from 19 countries across North America, Europe, and Asia. Among them, 19 teams from 23 organizations submitted their final results before the deadline. Finally, a joint team from IBM/INRIA and ENS de Lyon/Cornell Tech/Bar-ILan University won Track 1, CEA France won Track 2 and Seoul National University/UC San Diego won Track 3.

This special issue of BMC Medical Genomics highlights some most intriguing techniques reported during the competition.

Carpov et al. [4] describe their winning SGX based secure GWAS solution, which includes two key components i.e., (1) genome data compression and encryption and (2) top K most significant SNPs computation. Rust programming framework was used to enable massively parallel computation, which allows for the application to scale well with large input VCF files. It took about $1 \mathrm{~min}$ to handle the whole processing steps for about 30GB inputs.

(c) The Author(s). 2018 Open Access This article is distributed under the terms of the Creative Commons Attribution 4.0 International License (http://creativecommons.org/licenses/by/4.0/), which permits unrestricted use, distribution, and reproduction in any medium, provided you give appropriate credit to the original author(s) and the source, provide a link to the Creative Commons license, and indicate if changes were made. The Creative Commons Public Domain Dedication waiver (http://creativecommons.org/publicdomain/zero/1.0/) applies to the data made available in this article, unless otherwise stated. 
Laud et al. [5] report the results of using secure multiparty computation to efficiently solve privacy-preserving record linkage problem in large databases. The solution is built upon a commercial platform named Sharemind. To deduplicate 10 million record across 1000 different databases, it took about $30 \mathrm{~min}$ for computing servers over $100 \mathrm{Mbits} / \mathrm{s}$ network.

Kim et al. [6] present a winning solution for the homomorphic encryption based secure logistic regression. This solution is built upon an efficient approximate arithmetic homomorphic encryption library named HAEEN [7]. The experimental results show that a logistic regression training task over a dataset with 1,579 samples and 18 features can be finished within $6 \mathrm{~min}$.

Chen et al. [8] present another novel solution to the iDASH homomorphic encryption based secure logistic regression task. In their solution, they applied a multi-bit plaintext space in fully homomorphic encryption together with fixed point number encoding. Bootstrapping is combined in fully homomorphic encryption with a scaling operation in the fixed point arithmetics. They also use a minimax polynomial approximation to the sigmoid function and a 1-bit gradient descent method to reduce the plaintext growth in the training process. Their training over encrypted data took $0.4-3.2 \mathrm{~h}$ per iteration of the gradient descent.

Bonte et al. [9] discuss an alternative solution for secure logistic regression training over homomorphically encrypted data. The key idea of the proposed solution is based on a simplified fixed Hessian method of a much lower multiplicative complexity, which can be efficiently and iteratively solved under homomorphic operations.

All these new techniques showcase the achievements of this year's competition. Some of them have already demonstrated the potential of practical use, particularly the deduplication techniques for GA4GH, while the others report exciting progress that can lead to breakthroughs in genome privacy, including truly scalable and privacy-preserving genome analysis on untrusted cloud, based upon the new SGX hardware, and the feasibility of training classification models over fully encrypted data. All such techniques and findings will contribute to the genome-privacy research and move the science in this emerging domain forward.

\footnotetext{
About this supplement

This article has been published as part of BMC Medical Genomics Volume 11 Supplement 4, 2018: Proceedings of the 6th iDASH Privacy and Security Workshop 2017. The full contents of the supplement are available online at https://bmcmedgenomics.biomedcentral.com/articles/supplements/volume11-supplement-4.
}

\section{Authors' contributions}

$X W, H T, S W, X J$ designed the competition tasks and wrote the manuscript. WW, DB, LW, YJ, CW discussed the results and evaluated the performance for each track. All authors have read and approved the final manuscript.
Ethics approval and consent to participate

Not applicable.

\section{Competing interests}

The authors declare that they have no competing interests.

\section{Publisher's Note}

Springer Nature remains neutral with regard to jurisdictional claims in published maps and institutional affiliations.

\section{Author details}

${ }^{1}$ School of Informatics, Computing and Engineering, Indiana University, Bloomington, IN 47408, USA. ${ }^{2}$ UCSD Health Department of Biomedical Informatics, University of California San Diego, La Jolla, CA 92093, USA. ${ }^{3}$ School of Biomedical Informatics, The University of Texas Health Science Center, Houston, TX 77030, USA.

\section{Published: 11 October 2018}

\section{References}

1. iDASH Privacy \& security workshop 2017 - Home [lnternet]. [cited 2018 Apr 30]. Available from: http://humangenomeprivacy.org/2017

2. Trends D. DNA link to Golden State Killer raises questions of privacy versus safety. Fox News; 2018 [cited 2018 Apr 30]; Available from: http://www. foxnews.com/tech/2018/04/30/dna-link-to-golden-state-killer-raisesquestions-privacy-versus-safety.html

3. Samani SS, Huang Z, Ayday E, Elliot M, Fellay J, Hubaux J-P, et al. Quantifying genomic privacy via inference attack with high-order SNV correlations. Security and Privacy Workshops (SPW), 2015 IEEE. IEEE. 2015:32-40.

4. Sergiu Carpov TT. Secure top most significant genome variants search: iDASH 2017 competition. BMC Med Genomics. 2018;11(Suppl 4) https://doi.org/10.1186/s12920-018-0401-7.

5. Peeter Laud AP. Privacy-preserving record linkage in large databases using secure multiparty computation. BMC Med Genomics. 2018;11(Suppl 4) https://doi.org/10.1186/s12920-018-0400-8.

6. Andrey Kim, Yongsoo Song, Miran Kim, Keewoo Lee, Jung Hee Cheon. Logistic regression model training based on the approximate homomorphic encryption. BMC Med Genomics. 2018;11(Suppl 4) https://doi.org/10.1186/ s12920-018-0399-x

7. Cheon JH, Kim A, Kim M, Song Y. Homomorphic encryption for arithmetic of approximate numbers [Internet]. IACR Cryptology ePrint Archive. 2016; 421:2016. Available from: http://eprint.iacr.org/2016/421.pdf

8. Hao Chen, Ran Gilad-Bachrach, Kyoohyung Han, Zhicong Huang, Amir Jalali, Kim Laine, Kristin Lauter. Logistic regression over encrypted data from fully homomorphic encryption. BMC Med Genomics. 2018;11(Suppl 4) https://doi.org/10.1186/s12920-018-0397-z

9. Charlotte Bonte FV. Privacy-preserving logistic regression training. BMC Med Genomics. 2018;11(Suppl 4) https://doi.org/10.1186/s12920-018-0398-y.
Ready to submit your research? Choose BMC and benefit from:
- fast, convenient online submission
- thorough peer review by experienced researchers in your field
- rapid publication on acceptance
- support for research data, including large and complex data types
- gold Open Access which fosters wider collaboration and increased citations
- maximum visibility for your research: over $100 \mathrm{M}$ website views per year
At BMC, research is always in progress.
Learn more biomedcentral.com/submissions 